\title{
The existence of solutions to a Volterra integral equation
}

\author{
by Wojciech MydlarczyK (Wrocław)
}

\begin{abstract}
We study the equation $u=k * g(u)$ with $k$ such that $\ln k$ is convex or concave and $g$ is monotonic. Some necessary and sufficient conditions for the existence of nontrivial continuous solutions $u$ of this equation are given.
\end{abstract}

1. Introduction. We study the integral equation

$$
u(x)=\int_{0}^{x} k(x-s) g(u(s)) d s \quad(x>0)
$$

where

(i) $g:[0, \infty) \rightarrow[0, \infty)$ is continuous, nondecreasing, $g(0)=0$ and $x / g(x) \rightarrow 0$ as $x \rightarrow 0+$;

(ii) $k:(0, \infty) \rightarrow[0, \infty)$ is continuous, $\int_{0}^{\delta} k(s) d s<\infty$ and $\ln k$ is convex or concave.

In the sequel, we write $K(x)=\int_{0}^{x} k(s) d s$. We are interested in continuous, nonnegative and nontrivial, i.e. $u \neq \equiv 0$, solutions of (1.1). The existence of such solutions depends only on the behaviour of $k$ and $g$ near the origin. Therefore the conditions (i)-(ii) could be reformulated to take this fact into account.

There exists a wide literature (see [1], [4], [5], [6]) devoted to the equation (1.1). It is known that it always has a maximal solution (see [3]). If this maximal solution is $u \equiv 0$, then there are no other solutions. In the other case we observe the existence of infinitely many solutions, but each of them is either identically zero or a shifted maximal solution $u_{\delta}$ such that $u_{\delta}(x)=0$ for $x \in[0, \delta)$ and $u_{\delta}(x)=u(x-\delta)$ for $x \geq \delta$ (see [6]).

The maximal solution $u$ is a nondecreasing, absolutely continuous function, for which after integration by parts of the integral in (1.1) we obtain

1991 Mathematics Subject Classification: Primary 45D05.

Key words and phrases: the Volterra convolution type integral equations. 
a very useful relation

$$
u(x)=\int_{0}^{x} K(x-s) d g(u(s)), \quad x>0 .
$$

The basic role in the investigation of the existence problem for (1.1) is played by the following sufficient condition (see [3], [6]):

THEOREM 1.1. If

$$
w(x) \leq \int_{0}^{x} k(x-s) g(w(s)) d s, \quad x \geq 0,
$$

for some continuous function $w$ with $w(x)>0$ for $x>0$, then the equation (1.1) has a nontrivial maximal solution.

A very useful tool is also provided by the following comparison rule for (1.1) (see [2]):

THEOREM 1.2. Let functions $k_{i}$ and $g_{i}, i=1,2$, satisfy the assumptions (i)-(ii) and $k_{1} \leq k_{2}, g_{1} \leq g_{2}$. If $u_{1}$ and $u_{2}$ are the maximal solutions of (1.1) corresponding to the pairs $\left(k_{i}, g_{i}\right), i=1,2$, respectively, then

$$
u_{1}(x) \leq u_{2}(x) \quad \text { for } x>0 .
$$

In view of (ii), the function $k$ is monotonic near the origin. Define $c=$ $\lim _{x \rightarrow 0+} k(x)$.

If $0<c<\infty$, then we take $c_{1}<c<c_{2}$ and $k_{1}(x) \equiv c_{1}, k_{2}(x) \equiv c_{2}$. Consider the equations

$$
u(x)=c_{i} \int_{0}^{x} g(u(s)) d s \quad(x>0, i=1,2) .
$$

By differentiation of (1.3) we can easily check that the condition

$$
\int_{0}^{\delta} \frac{d s}{g(s)}<\infty \quad(\delta>0)
$$

is sufficient and necessary for the existence of nontrivial solutions to (1.3). Now, using comparison arguments and Theorem 1.1 we can see that (1.4) is also necessary and sufficient for the existence of nontrivial solutions to (1.1) in the considered case.

The cases $c=0$ and $c=\infty$ require other arguments. First we observe that in view of the assumed convexity or concavity of $\ln k$ it follows that if $c=0$, then $\ln k$ is nondecreasing and if $c=\infty$, then $\ln k$ is nonincreasing. So, we assume in addition that ing.

(iii) $\ln k$ is either concave and nondecreasing, or convex and nonincreas- 
By differentiation we can easily check

R e m a r k 1.1. The function $\ln k$ is convex (concave) if and only if $k \circ K^{-1}$ is convex (concave), where $K^{-1}$ is the inverse function to $K$.

In the next section we give some a priori estimates of the maximal solution to (1.1) which play a basic role in the construction of necessary and sufficient conditions for the existence of nontrivial solutions to (1.1).

The same problems were considered in [5] under an additional assumption that $g$ is absolutely continuous. In this paper we obtain the same necessary and sufficient conditions, but in a simpler way and without that assumption.

2. Main results. We begin with proving a priori estimates for a nontrivial maximal solution of (1.1).

TheOREM 2.1. Let the conditions (i)-(iii) be satisfied. Then the nontrivial maximal solution $u$ of (1.1) can be estimated as follows:

$$
\begin{aligned}
\int_{0}^{x}\left(K^{-1}\right)^{\prime}\left(\frac{s}{g(s)}\right) & \frac{d s}{g(s)} \\
& \leq u^{-1}(x) \leq K^{-1}\left(\frac{x}{g(x)}\right)+\int_{0}^{x} K^{-1}\left(\frac{s}{g(s)}\right) \frac{d s}{g(s)}
\end{aligned}
$$

if $\ln k$ is concave and nondecreasing, and

$$
\begin{aligned}
K^{-1}\left(\frac{x}{g(x)}\right)+\int_{0}^{x} K^{-1}\left(\frac{s}{g(s)}\right) & \frac{d s}{g(s)} \\
& \leq u^{-1}(x) \leq \int_{0}^{x}\left(K^{-1}\right)^{\prime}\left(\frac{s}{g(s)}\right) \frac{d s}{g(s)}
\end{aligned}
$$

if $\ln k$ is convex and nonincreasing; $u^{-1}$ is the inverse function to $u$.

Pr o of. We consider the case where $\ln k$ is concave and nondecreasing. The other case can be considered similarly.

The proof of the left inequality is short and it can be found for example in [4]. For the reader's convenience, we present it also below. Since $k=$ $k \circ K^{-1} \circ K$ and $k \circ K^{-1}$ is concave, we can apply the Jensen inequality to the relation

$$
u^{\prime}(x)=\int_{0}^{x} k(x-s) d g(u(s))
$$

obtained from (1.2), which gives the inequality

$$
u^{\prime}(x) \leq k \circ K^{-1}\left(\frac{u(x)}{g(u(x))}\right) g(u(x)),
$$


and further

$$
\left(K^{-1}\right)^{\prime}\left(\frac{u(x)}{g(u(x))}\right) \frac{u^{\prime}(x)}{g(u(x))} \leq 1 .
$$

Now, by integration we obtain the left inequality in (2.1).

We begin the proof of the right inequality with the observation that $K$ is convex. By applying the Jensen inequality to (1.2) we get

$$
u(x) \geq g(u(x)) K\left(\frac{\int_{0}^{x}(x-s) d g(u(s))}{g(u(s))}\right) .
$$

Note that $\int_{0}^{x}(x-s) d g(u(s))=\int_{0}^{x} g(u(s)) d s$ and define

$$
V(x)=\frac{\int_{0}^{x} g(u(s)) d s}{g(u(x))} \quad \text { for } x>0 \text { and } V(0)=0 .
$$

From (2.3) we get

$$
V(x) \leq K^{-1}\left(\frac{u(x)}{g(u(x))}\right) .
$$

The function $V$ is continuous and of bounded variation on every interval away from zero. We can easily see that

$$
d V(x)=d x-V(x) \frac{d g(u(x))}{g(u(x))} \quad(x>0) .
$$

Hence and from (2.4) we get

$$
\begin{aligned}
x & =\lim _{\delta \rightarrow 0+}\left(\int_{\delta}^{x} d V(s)+\int_{\delta}^{x} V(s) \frac{d g(u(s))}{g(u(s))}\right) \\
& \leq K^{-1}\left(\frac{u(x)}{g(u(x))}\right)+\int_{0}^{x} K^{-1}\left(\frac{u(s)}{g(u(s))}\right) \frac{d g(u(s))}{g(u(s))} \quad(x>0),
\end{aligned}
$$

from which our assertion follows.

As a consequence of the left inequalities in (2.1) and (2.2) we obtain a necessary condition for the existence of a nontrivial solution of (1.1). It is stated in

Theorem 2.2. Let the conditions (i)-(iii) be satisfied. Then the conditions:

$$
\int_{0}^{\delta}\left(K^{-1}\right)^{\prime}\left(\frac{s}{g(s)}\right) \frac{d s}{g(s)}<\infty \quad(\delta>0),
$$

if $\ln k$ is concave and nondecreasing, and

$$
\int_{0}^{\delta} K^{-1}\left(\frac{s}{g(s)}\right) \frac{d g(s)}{g(s)}<\infty \quad(\delta>0),
$$


if $\ln k$ is convex and nonincreasing, are necessary for the existence of nontrivial solutions of (1.1).

A sufficient condition for the existence of nontrivial solutions of (1.1) can be established on the basis of the right inequalities in (2.1) and (2.2). It is stated in

Theorem 2.3. Let the conditions (i)-(iii) be satisfied. Then the conditions:

$$
\int_{0}^{\delta} K^{-1}\left(\frac{s}{g(s)}\right) \frac{d g(s)}{g(s)}<\infty \quad(\delta>0),
$$

if $\ln k$ is concave and nondecreasing, and

$$
\int_{0}^{\delta}\left(K^{-1}\right)^{\prime}\left(\frac{s}{g(s)}\right) \frac{d s}{g(s)}<\infty \quad(\delta>0),
$$

if $\ln k$ is convex and nonincreasing, are sufficient for the existence of nontrivial solutions of (1.1).

Proof. In view of Theorem 1.1 it suffices to construct a function $w$ such that the corresponding inequality is satisfied. If $w$ is increasing, then the substitution $\tau=w(s)$ shows that the inequality can be written in the equivalent form

$$
x \leq \int_{0}^{x} K\left(w^{-1}(x)-w^{-1}(\tau)\right) d g(\tau),
$$

where $w^{-1}$ is the inverse function to $w$.

We are going to construct a nondecreasing function satisfying (2.6). First we consider the case where $\ln k$ is concave and nondecreasing. In view of our assumptions the function

$$
F(x)=K^{-1}\left(\frac{x}{g(x)}\right)+\int_{0}^{x} K^{-1}\left(\frac{s}{g(s)}\right) \frac{d g(s)}{g(s)} \quad \text { for } x>0
$$

and $F(0)=0$ is well defined. The function $\Psi(x)=K^{-1}(x / g(x))$ for $x>0$ and $\Psi(0)=0$ is continuous and since $K^{-1}$ is sufficiently smooth, it is of bounded variation on every interval away from zero. Furthermore, we can easily check that

$$
d \Psi(x)=\left(K^{-1}\right)^{\prime}\left(\frac{x}{g(x)}\right) \frac{d x}{g(x)}-\frac{x}{g(x)}\left(K^{-1}\right)^{\prime}\left(\frac{x}{g(x)}\right) \frac{d g(x)}{g(x)} .
$$

Since $K$ is convex, $K^{-1}$ is concave, which implies that $z\left(K^{-1}\right)^{\prime}(z) \leq$ 
$K^{-1}(z)$ for $z>0$. Hence, we obtain

$$
\int_{0}^{x} \frac{s}{g(s)}\left(K^{-1}\right)^{\prime}\left(\frac{s}{g(s)}\right) \frac{d g(s)}{g(s)} \leq \int_{0}^{x} K^{-1}\left(\frac{s}{g(s)}\right) \frac{d g(s)}{g(s)} .
$$

Now, we observe that

$$
\begin{aligned}
F(x)= & \lim _{\delta \rightarrow 0+}\left(\int_{\delta}^{x} d \Psi(s)+\int_{\delta}^{x} K^{-1}\left(\frac{s}{g(s)}\right) \frac{d g(s)}{g(s)}\right) \\
= & \int_{0}^{x}\left(K^{-1}\right)^{\prime}\left(\frac{s}{g(s)}\right) \frac{d s}{g(s)} \\
& +\int_{0}^{x}\left(K^{-1}\left(\frac{s}{g(s)}\right)-\frac{s}{g(s)}\left(K^{-1}\right)^{\prime}\left(\frac{s}{g(s)}\right)\right) \frac{d g(s)}{g(s)},
\end{aligned}
$$

from which it follows that $F$ is increasing. We will check that $F$ satisfies (2.6), so $F^{-1}$ can be taken as the required $w$.

Define

$$
\left.\Phi(x)=\int_{0}^{x} K(F(x)-F(s))\right) d g(s),
$$

Now applying the Jensen inequality we get

$$
\Phi(x) \geq g(x) K\left(\frac{\int_{0}^{x}(F(x)-F(s)) d g(s)}{g(x)}\right) .
$$

Noting that $\int_{0}^{x}(F(x)-F(s)) d g(s)=\int_{0}^{x} g(s) d F(s)$ and $g(s) d F(s)=$ $d\left[g(s) K^{-1}(s / g(s))\right]$, from (2.7) we immediately get $\Phi(x) \geq x$, which ends the proof in this case.

If $\ln k$ is convex and nonincreasing, we define

$$
F(x)=\int_{0}^{x}\left(K^{-1}\right)^{\prime}\left(\frac{s}{g(s)}\right) \frac{d s}{g(s)}, \quad x>0 .
$$

As previously, we will check that $F$ satisfies (2.6).

Define

$$
\Phi(x)=\int_{0}^{x} K(F(x)-F(s)) d g(s), \quad \Phi(0)=0 .
$$

Note that

$$
\begin{aligned}
\Phi(x) & =\int_{0}^{x}\left[\int_{s}^{x} F^{\prime}(v) k(F(v)-F(s)) d v\right] d g(s) \\
& =\int_{0}^{x}\left[F^{\prime}(v) \int_{0}^{v} k(F(v)-F(s)) d g(s)\right] d v .
\end{aligned}
$$


Hence $\Phi$ is absolutely continuous and

$$
\Phi^{\prime}(x)=F^{\prime}(x) \int_{0}^{x} k(F(x)-F(s)) d g(s) \quad \text { a.e. }
$$

Since $k=k \circ K^{-1} \circ K$ and $k \circ K^{-1}$ is convex, we can apply the Jensen inequality to obtain

$$
\Phi^{\prime}(x) \geq F^{\prime}(x) g(x) k \circ K^{-1}\left(\frac{\Phi(x)}{g(x)}\right)=\frac{k \circ K^{-1}(\Phi(x) / g(x))}{k \circ K^{-1}(x / g(x))} .
$$

Our aim is to prove that $\Phi(x) \geq x$ for $x>0$. Assume that $\Phi\left(x_{0}\right)<x_{0}$ for some $x_{0}>0$. Now, let $0 \leq x_{1}<x_{0}$ be chosen so that

$$
x_{1}=\Phi\left(x_{1}\right) \quad \text { and } \Phi(x)<x \quad \text { for } x \in\left(x_{1}, x_{0}\right) .
$$

Since $k$ is nonincreasing and $K^{-1}$ is nondecreasing, from (2.8) we get

$$
\Phi^{\prime}(x) \geq 1 \quad \text { for } x \in\left(x_{1}, x_{0}\right) .
$$

Hence $\Phi\left(x_{0}\right)-\Phi\left(x_{1}\right) \geq x_{0}-x_{1}$, which contradicts $\Phi\left(x_{0}\right)<x_{0}$ and ends the proof.

For some class of functions $k$, one can replace $\int_{0}^{\delta} K^{-1}(s / g(s)) \frac{d g(s)}{g(s)}$ with $\int_{0}^{\delta} K^{-1}(s / g(s)) \frac{d s}{s}$, which is usually easier to compute. Namely, we have

Remark 2.1. If $\int_{0}^{\delta} K^{-1}(s) \frac{d s}{s}<\infty(\delta>0)$, then the integrals

$$
\int_{0}^{\delta} K^{-1}\left(\frac{s}{g(s)}\right) \frac{d g(s)}{g(s)} \text { and } \int_{0}^{\delta} K^{-1}\left(\frac{s}{g(s)}\right) \frac{d s}{s}
$$

are simultaneously convergent or divergent.

Pro of. Let $\Phi(x)=\int_{0}^{x} K^{-1}(s) \frac{d s}{s}$. Then the function $\Psi(x)=\Phi(x / g(x))$ for $x>0$ and $\Psi(0)=0$ is continuous and of bounded variation on every interval away from zero. Furthermore, we have

$$
d \Phi\left(\frac{x}{g(x)}\right)=K^{-1}\left(\frac{x}{g(x)}\right) \frac{d x}{x}-K^{-1}\left(\frac{x}{g(x)}\right) \frac{d g(x)}{g(x)} \quad(x>0) .
$$

Now, our assertion follows from the equality

$$
\Phi(\delta / g(\delta))=\lim _{a \rightarrow 0+} \int_{a}^{\delta} d \Phi(s / g(s)) .
$$

EXAMPLE. Consider the equation

$$
u(x)=\int_{0}^{x}(x-s)^{\alpha-1} g(u(s)) d s \quad(x, \alpha>0) .
$$


In [3] it was shown that if $g$ satisfies (i) then the condition

$$
\int_{0}^{\delta}\left[\frac{s}{g(s)}\right]^{1 / \alpha} \frac{d s}{s}<\infty \quad(\delta>0)
$$

is necessary and sufficient for the existence of nontrivial solutions of (2.9).

We now show how this result follows from Theorems 2.2 and 2.3.

In the considered case we have $k(x)=x^{\alpha-1}, K(x)=\frac{1}{\alpha} x^{\alpha}$ and $K^{-1}(x)=$ $\alpha^{1 / \alpha} x^{1 / \alpha}$. For $0<\alpha \leq 1, \ln k$ is convex and nonincreasing, and for $\alpha>$

1 , it is concave and nondecreasing. Since $\int_{0}^{\delta} K^{-1}(s) \frac{d s}{s}<\infty$, in view of Remark 2.1 and Theorems 2.2 and 2.3 we have to consider the integrals

$$
\int_{0}^{\delta}\left(K^{-1}\right)^{\prime}\left(\frac{s}{g(s)}\right) \frac{d s}{g(s)} \text { and } \int_{0}^{\delta} K^{-1}\left(\frac{s}{g(s)}\right) \frac{d s}{s} .
$$

But they are of the same form as the integral in (2.10), which gives the required assertion.

\section{References}

[1] G. Gripenberg, On the uniqueness of solutions of Volterra equations, J. Integral Equations Appl. 2 (1990), 421-430.

[2] R. K. Miller, Nonlinear Volterra Integral Equations, W. A. Benjamin, Menlo Park, Calif., 1971.

[3] W. Mydlarczyk, The existence of nontrivial solutions of Volterra equations, Math. Scand. 68 (1991), 83-88.

[4] -, Remarks on a nonlinear Volterra equation, Ann. Polon. Math. 53 (1991), 227-232.

[5] W. Okrasiński, Nontrivial solutions to nonlinear Volterra integral equations, SIAM J. Math. Anal. 4 (1991), 1007-1015.

[6] -, On a nonlinear Volterra integral equation, Math. Methods Appl. Sci. 8 (1986), $345-350$

Mathematical Institute

University of Wrocław

pl. Grunwaldzki $2 / 4$

50-384 Wrocław, Poland

E-mail: mydlar@math.uni.wroc.pl 